\title{
Determinação de regiões pluviométricas homogêneas na bacia hidrográfica do rio Piquiri - Paraná
}

\author{
Paulo Miguel de Bodas Terassi ${ }^{\text {(a) }}$, Márcio Greyck Guimarães Correa ${ }^{(b)}$, Emerson Galvani ${ }^{(c)}$ \\ (a) Doutorando em Geografia Física, Universidade de São Paulo, pmbterassi@ usp.br \\ (b) Doutorando em Geografia Física, Universidade de São Paulo, mgreyckcorrea@usp.br \\ (c) Professor Doutor do Departamento de Geografia, Universidade de São Paulo, egalvani@usp.br
}

Eixo: Climatologia em diferentes níveis escalares - Mudanças e variabilidades

\begin{abstract}
Resumo
Este trabalho objetiva delimitar as regiões pluviométricas homogêneas tendo com base a análise de agrupamento para a bacia hidrográfica do rio Piquiri - Paraná. Utilizaram-se os dados de quarenta postos pluviométricos administrados pelo Instituto das Águas do Paraná para o período de 1976 a 2015. O principal procedimento metodológico utilizado neste trabalho corresponde à técnica de análise cluster, com a escolha da medida de proximidade a distância Euclidiana e do método hierárquico de agrupamento Ward. Mostrou-se que a área em estudo apresenta duas regiões pluviométricas distintas: o grupo homogêneo I apresenta menores totais pluviais devido às menores altitudes e, com uma sazonalidade marcada, característica do clima tropical; o grupo homogêneo II mostrou os totais pluviométricos mais elevados, o que se deve às maiores altitudes e ao predomínio do clima subtropical.
\end{abstract}

Palavras chave: precipitação pluvial, análise de agrupamento, bacia hidrográfica.

\section{Introdução}

A pluviosidade é o atributo climático de maior impacto e interação na dinâmica ambiental das bacias hidrográficas em regiões de clima tropical interferindo diretamente na estrutura e funcionamento do ciclo hidrológico (SANTOS; ARAÚJO, 2013). Devido às características próprias ao clima tropical, observa-se a predominância de elevados totais pluviométricos no território brasileiro, exceção dada pela região do semiárido nordestino (ÁLVARES et al., 2013).

Melo Júnior et al. (2006) destacam que o estabelecimento de metodologias apropriadas na definição do regime de pluviosidade em níveis regionais é essencial para a tomada de decisões do uso dos recursos hídricos. Uma das técnicas mais utilizadas para analisar a distribuição espacial das chuvas é a técnica de análise de agrupamento, que faz parte de um conjunto de técnicas estatísticas denominadas de Análise Multivariada, e comumente utilizada para agrupar objetos para formar grupos homogêneos internamente e heterogêneos entre si (MARTIN et al., 2008; SILVESTRE et al., 2013). 
Keller Filho et al. (2006) identificaram vinte e cinco regiões pluviométricas homogêneas para o território brasileiro a partir da utilização da análise de agrupamento hierárquica, formando grupos de observações similares quanto ao regime de chuva. Estes autores destacaram que a identificação de regiões homogêneas quanto à distribuição pluviométrica é fundamental para a definição do calendário agrícola, uma vez que o detalhamento dos padrões pluviais auxilia na escolha de culturas de menor risco e para o estabelecimento das datas de plantio mais favoráveis.

Fritzsons et al. (2011) obtiveram três grandes grupos quanto ao regime de pluviosidade para o Paraná: o primeiro na região Norte, de significativa redução da precipitação pluvial nos meses de inverno, com características mais aproximadas ao clima tropical do Brasil Central; o segundo no setor centro-sul e leste do Estado, com os maiores totais pluviométricos anuais e de homogeneidade na distribuição mensal, inerente ao domínio do clima subtropical do Brasil Meridional; o terceiro no setor central, sudeste e norte, com características intermediárias em relação ao dois grande grupos citados anteriormente, ou seja, com menores totais anuais de pluviosidade em relação ao segundo grupo e de menor concentração das chuvas no período do verão em relação ao primeiro grupo.

Com a finalidade de compreender a variabilidade pluviométrica e sua relação com a dinâmica atmosférica na bacia hidrográfica do rio Ivaí (PR), Baldo (2006) empregou a técnica da análise de agrupamento para a área referida e obteve quatro grupos homogêneos em relação ao padrão do regime de precipitação e destacou a importância de compreender as características topográficas para a determinação do ponto de corte dendograma, posto que o desempenho da orografia para o controle espacial de maior ou menor pluviosidade. Concordantes com a observação supracitada sobre o controle orográfico, Chierice e Landim (2014) utilizaram-se da análise de agrupamento para analisar a variabilidade das chuvas na bacia hidrográfica do rio Mogi Guaçu e obtiveram maiores totais pluviométricos para os grupos homogêneos situados nas maiores altitudes e, inversamente, a redução das chuvas nos grupos homogêneos nos setores de menores altitudes.

A área de estudo do presente trabalho corresponde à bacia hidrográfica do rio Piquiri (Figura 1), localizada no setor centro-oeste do Estado do Paraná, entre as latitudes $23^{\circ} 00^{\prime} \mathrm{S}$ e $25^{\circ} 00^{\prime} \mathrm{S}$ e as longitudes

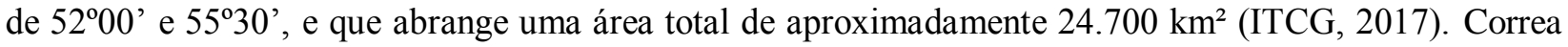
(2013) observou que a bacia hidrográfica supracitada caracteriza-se pelos maiores totais anuais de pluviosidade para o setor centro-sul, com valores superiores a $2.000 \mathrm{~mm}$, devido à influência da altitude e a dinâmica atmosférica regional, com uma maior influência dos mecanismos extratropicais para a geração de chuvas. Inversamente, o setor norte da bacia hidrográfica apresenta pluviosidade média anual próxima a $1.400 \mathrm{~mm}$, condição atribuída às menores altitudes e a proximidade com o domínio do clima tropical. 


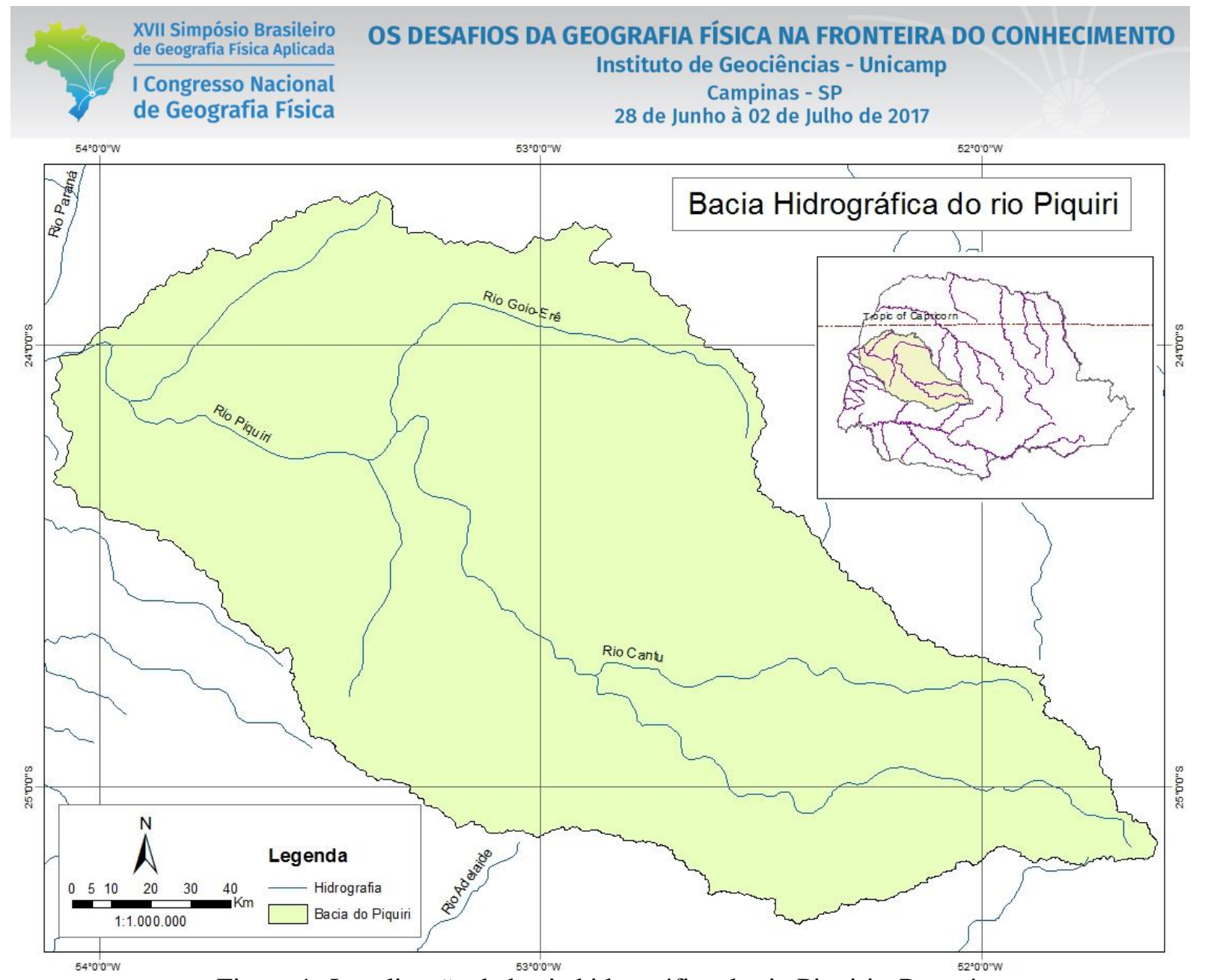

Figura 1- Localização da bacia hidrográfica do rio Piquiri - Paraná.

O estudo anterior de Correa (2013) aplicou técnica da krigagem para a interpolação espacial dos dados pluviométricos e, portanto, a utilização da técnica de agrupamento empregada neste trabalho possibilitou uma diferente análise espacial da precipitação pluvial. Compreendendo a importância do estudo da distribuição espaço-temporal pluviométrica para a área em estudo e a aplicação da técnica discorrida, objetiva-se delimitar as regiões pluviométricas homogêneas tendo como base a análise de agrupamento na bacia hidrográfica do rio Piquiri.

\section{Materiais e Métodos}

Utilizaram-se os dados de quarenta postos pluviométricos administrados pelo Instituto das Águas do Paraná e que compreenderam a série histórica de 1976 a 2015 (Quadro I). Estes dados de precipitação pluvial foram trabalhados a partir da utilização da planilha eletrônica Microsoft Office Excel 2007, na qual se averiguaram a ausência de registros, que foram inferiores a 5\%. Os dados faltantes dos postos pluviométricos foram preenchidos seguindo o método de ponderação regional apresentado por Villela e Mattos (1975), que tem como base o registro pluviométrico de três postos localizados o mais próximo 
possível do posto em que se verifica a falta de dados, com características pluviométricas e altitudes semelhantes. Leivas et al. (2006) esclarecem que os registros faltantes de pluviosidade neste método são estimados pela média ponderada do registro das três estações vizinhas, onde os pesos são as razões entre as precipitações normais anuais.

Quadro I - Localização geográfica dos postos pluviométricos na bacia hidrográfica do rio Piquiri - Paraná.

\begin{tabular}{|c|c|c|c|c|c|}
\hline $\mathbf{N}^{\mathbf{o}}$ & Postos Pluviométricos & Long. $\left({ }^{\circ} \mathrm{S}\right)$ & Lat. $\left({ }^{\circ} \mathbf{W}\right)$ & Altitude (m) & Código ANEEL \\
\hline 1 & Altamira do Paraná & $52^{\circ} 42^{\prime} 00^{\prime \prime}$ & $24^{\circ} 48^{\prime} 00^{\prime \prime}$ & 650 & 2452012 \\
\hline 2 & Altamira do Paraná (Balsa do Cantu) & $52^{\circ} 42^{\prime} 00^{\prime}$ & $24^{\circ} 45^{\prime} 00^{\prime \prime}$ & 360 & 2452000 \\
\hline 3 & Alto Piquiri & $53^{\circ} 19^{\prime} 59^{\prime}{ }^{\prime}$ & $24^{\circ} 12^{\prime} 00^{\prime \prime}$ & 427 & 2453008 \\
\hline 4 & Alto Piquiri (Porto Formosa) & $53^{\circ} 26^{\prime} 17^{\prime \prime}$ & $24^{\circ} 01^{\prime} 48^{\prime \prime}$ & 430 & 2453009 \\
\hline 5 & Assis Chateaubriand & $53^{\circ} 36^{\prime} 51^{\prime \prime}$ & $24^{\circ} 36^{\prime} 40^{\prime \prime}$ & 501 & 2453030 \\
\hline 6 & Brasilândia do Sul & $53^{\circ} 31^{\prime} 32^{\prime \prime}$ & $24^{\circ} 11^{\prime} 54^{\prime \prime}$ & 396 & 2453050 \\
\hline 7 & Cafezal do Sul & $53^{\circ} 35^{\prime} 11^{\prime \prime}$ & $23^{\circ} 56^{\prime} 29^{\prime \prime}$ & 383 & 2353047 \\
\hline 8 & Campina da Lagoa & $52^{\circ} 48^{\prime} 15^{\prime \prime}$ & $24^{\circ} 35^{\prime} 59^{\prime \prime}$ & 618 & 2452011 \\
\hline 9 & Campina da Lagoa (Ponte do Goio Bang) & $52^{\circ} 54^{\prime} 07^{\prime}$ & $24^{\circ} 34^{\prime} 53^{\prime \prime}$ & 320 & 2452001 \\
\hline 10 & Campina do Simão & $51^{\circ} 48^{\prime} 23^{\prime \prime}$ & $25^{\circ} 06^{\prime} 33^{\prime \prime}$ & 1056 & 2551009 \\
\hline 11 & Campo Bonito & $53^{\circ} 04^{\prime} 00^{\prime}{ }^{\prime}$ & $24^{\circ} 52^{\prime} 59^{\prime \prime}$ & 700 & 2453014 \\
\hline 12 & Cascavel & $53^{\circ} 14^{\prime} 36^{\prime \prime}$ & $24^{\circ} 57^{\prime} 43^{\prime \prime}$ & 662 & 2453056 \\
\hline 13 & Corbélia & $53^{\circ} 17^{\prime} 29^{\prime \prime}$ & $24^{\circ} 47^{\prime} 55^{\prime \prime}$ & 696 & 2453012 \\
\hline 14 & Farol & $52^{\circ} 37^{\prime} 17^{\prime \prime}$ & $24^{\circ} 05^{\prime} 26^{\prime \prime}$ & 582 & 2452029 \\
\hline 15 & Formosa do Oeste & $53^{\circ} 19^{\prime} 00^{\prime \prime}$ & $24^{\circ} 16^{\prime} 59^{\prime \prime}$ & 370 & 2453010 \\
\hline 16 & Francisco Alves & $53^{\circ} 57^{\prime} 52^{\prime \prime}$ & $24^{\circ} 05^{\prime} 42^{\prime \prime}$ & 302 & 2453052 \\
\hline 17 & Goioerê & $53^{\circ} 01^{\prime} 55^{\prime \prime}$ & $24^{\circ} 11^{\prime} 36^{\prime \prime}$ & 497 & 2453016 \\
\hline 18 & Guaraniaçú & $52^{\circ} 52^{\prime} 59^{\prime \prime}$ & $25^{\circ} 04^{\prime} 59^{\prime \prime}$ & 920 & 2552006 \\
\hline 19 & Iguatu & $53^{\circ} 06^{\prime} 00^{\prime \prime}$ & $24^{\circ} 37^{\prime} 59^{\prime \prime}$ & 730 & 2453013 \\
\hline 20 & Janiópolis & $52^{\circ} 46^{\prime} 00^{\prime \prime}$ & $24^{\circ} 07^{\prime} 59^{\prime \prime}$ & 350 & 2452010 \\
\hline 21 & Juranda & $52^{\circ} 55^{\prime} 57^{\prime \prime}$ & $24^{\circ} 20^{\prime} 46^{\prime \prime}$ & 513 & 2452033 \\
\hline 22 & Laranjal & $52^{\circ} 28^{\prime} 26^{\prime \prime}$ & $24^{\circ} 53^{\prime} 09^{\prime \prime}$ & 741 & 2452019 \\
\hline 23 & Laranjeiras do Sul & $52^{\circ} 25^{\prime} 52^{\prime \prime}$ & $25^{\circ} 13^{\prime} 21^{\prime \prime}$ & 785 & 2552019 \\
\hline 24 & Mamborê (Guarani) & $52^{\circ} 33^{\prime} 00^{\prime \prime}$ & $24^{\circ} 25^{\prime} 59^{\prime \prime}$ & 650 & 2452035 \\
\hline 25 & Mamborê (Pensamento) & $52^{\circ} 39^{\prime} 00^{\prime \prime}$ & $24^{\circ} 19^{\prime} 00^{\prime \prime}$ & 638 & 2452040 \\
\hline 26 & Mariluz & $53^{\circ} 07^{\prime} 00^{\prime \prime}$ & $23^{\circ} 55^{\prime} 00^{\prime \prime}$ & 345 & 2353006 \\
\hline 27 & Mariluz (Balsa do Goioerê) & $53^{\circ} 10^{\prime} 00^{\prime \prime}$ & $23^{\circ} 58^{\prime} 59^{\prime \prime}$ & 320 & 2353002 \\
\hline 28 & Maripá & $53^{\circ} 49^{\prime} 38^{\prime \prime}$ & $24^{\circ} 25^{\prime} 21^{\prime \prime}$ & 394 & 2453047 \\
\hline 29 & Marquinho & $52^{\circ} 15^{\prime} 30^{\prime \prime}$ & $25^{\circ} 06^{\prime} 44^{\prime \prime}$ & 872 & 2552008 \\
\hline 30 & Nova Aurora & $53^{\circ} 22^{\prime} 48^{\prime \prime}$ & $24^{\circ} 34^{\prime} 23^{\prime \prime}$ & 544 & 2453037 \\
\hline 31 & Nova Santa Rosa & $53^{\circ} 57^{\prime} 21^{\prime \prime}$ & $24^{\circ} 23^{\prime} 24^{\prime \prime}$ & 341 & 2453048 \\
\hline 32 & Palmital & $52^{\circ} 12^{\prime} 10^{\prime \prime}$ & 2453’04'” & 890 & 2452016 \\
\hline 33 & Pérola & $53^{\circ} 40^{\prime} 32^{\prime \prime}$ & $23^{\circ} 47^{\prime} 49^{\prime \prime}$ & 438 & 2353016 \\
\hline 34 & Roncador & $52^{\circ} 16^{\prime} 34^{\prime \prime}$ & $24^{\circ} 35^{\prime} 30^{\prime \prime}$ & 723 & 2452015 \\
\hline 35 & Santa Maria do Oeste & $51^{\circ} 57^{\prime} 16^{\prime \prime}$ & $24^{\circ} 46^{\prime} 45^{\prime \prime}$ & 900 & 2451010 \\
\hline 36 & Santa Maria do Oeste (Balsa Grande) & $51^{\circ} 52^{\prime} 59^{\prime \prime}$ & $24^{\circ} 55^{\prime} 59^{\prime \prime}$ & 929 & 2451021 \\
\hline 37 & Tuneiras do Oeste & $52^{\circ} 57^{\prime} 59^{\prime \prime}$ & $23^{\circ} 54^{\prime} 24^{\prime \prime}$ & 459 & 2352026 \\
\hline 38 & Ubiratã & $53^{\circ} 10^{\prime} 00^{\prime}$ & $24^{\circ} 31^{\prime} 00^{\prime \prime}$ & 509 & 2452009 \\
\hline 39 & Ubiratã (Porto Piquiri) & $52^{\circ} 59^{\prime} 25^{\prime \prime}$ & $24^{\circ} 32^{\prime} 00^{\prime \prime}$ & 310 & 2453001 \\
\hline 40 & Xambrê & $53^{\circ} 29^{\prime} 11^{\prime \prime}$ & $23^{\circ} 44^{\prime} 03^{\prime \prime}$ & 412 & 2353005 \\
\hline
\end{tabular}


O principal procedimento metodológico utilizado neste trabalho corresponde à técnica de análise cluster (agrupamento), utilizada com a finalidade de realizar uma análise detalhada e setorizada da área de estudo, delimitando regiões similares quanto ao padrão pluviométrico. A análise de agrupamento considera um conjunto inicial de objetos, no qual são associadas medidas de várias grandezas variáveis classificatórias, utilizadas para se obter grupos de objetos assemelhados em relação aos valores assumidos por essas variáveis (EVERRIT, 1993). Para tanto, considerou-se como principal aspecto os registros mensais de precipitação pluvial para os postos pluviométricos, considerado por Teodoro et al. (2015) e Brito et al. (2016) essencial para averiguar a relação entre os valores observados e a dinâmica atmosférica regional.

Utilizou-se como medida de proximidade a distância Euclidiana ao quadrado, comumente a mais utilizada para a análise de variáveis quantitativas e, segundo Freitas et al. (2013), corresponde a distância geométrica no espaço multidimensional entre duas observações dimensionais. Mello Júnior et al. (2006) a descrevem como a distância geométrica tomada em um espaço de p dimensões. Sendo $X_{i j}$ a observação da i-ésima estação pluviométrica $(i=1,2, \ldots, n)$, com referência a j-ésima variável ou frequência absoluta em cada classe $(j=1,2, \ldots, p)$ estudada, define-se a distância euclidiana padronizada entre dois postos i e i' por meio da expressão (Equação 1), em que $\mathrm{Z}_{\mathrm{ij}}, \mathrm{s}\left(\mathrm{X}_{\mathrm{j}}\right)$ e $\bar{X}$ são, respectivamente, a variável padronizada, o desvio-padrão e a média da j-ésima variável original (Equação 2).

$$
d_{i i^{J}}=\sqrt{\sum_{j=1}^{p}\left(z_{i j}-z_{i^{r}}\right)^{2}}(\text { Eq. } 1) \quad Z_{i j}=\frac{X_{i j}-X}{\delta\left(x_{j}\right)}(\text { Eq. } 2)
$$

Amplamente utilizado, usou-se o método Ward (1963) como o mais apropriado para a análise de agrupamento, pois propõe que em qualquer fase de análise a perda de informação que resulta do agrupamento de elementos seja medida pela soma dos quadrados dos desvios de cada elemento à média dos elementos do grupo à qual pertente (NASCIMENTO et al., 2015; WREGE et al., 2016).

Partindo-se dos dados mensais de pluviosidade, elaborou-se uma tabela do tipo "indivíduos" versus "variáveis", considerando-se como indivíduo cada um dos postos pluviométrico, e as variáveis como os totais pluviométricos mensais. A representação do dendograma foi gerada a partir do software Statistica 7.0 e realizou-se o ponto de corte a partir de uma análise subjetiva que considerou as características do relevo, o regime de pluviosidade e a proximidade espacial entre os postos pluviométricos.

\section{Resultados e discussões}

A figura 1 corresponde ao dendograma da técnica de análise de agrupamento dos postos pluviométricos da bacia hidrográfica do rio Piquiri - Paraná. A aplicação deste procedimento indicou que a área em estudo 




apresenta dois grandes grupos pluviométricos, subdivididos em três subgrupos (Ia, Ib e Ic) para o grupo homogêneo I e dois subgrupos (IIa e IIb) para o grupo homogêneo II.

A técnica de agrupamento permite a delimitação de regiões com similaridade no padrão pluviométrico, possibilitando identificar a tipologia pluvial da área de estudo e demonstrar associações com as características do relevo e da circulação atmosférica. O critério determinante para a realização do ponto de corte do dendograma foi o relevo, considerando-se que os postos pluviométricos do grupo homogêneo I situam-se a jusante do curso principal do rio Piquiri e, inversamente, o grupo homogêneo II localiza-se no setor a montante.

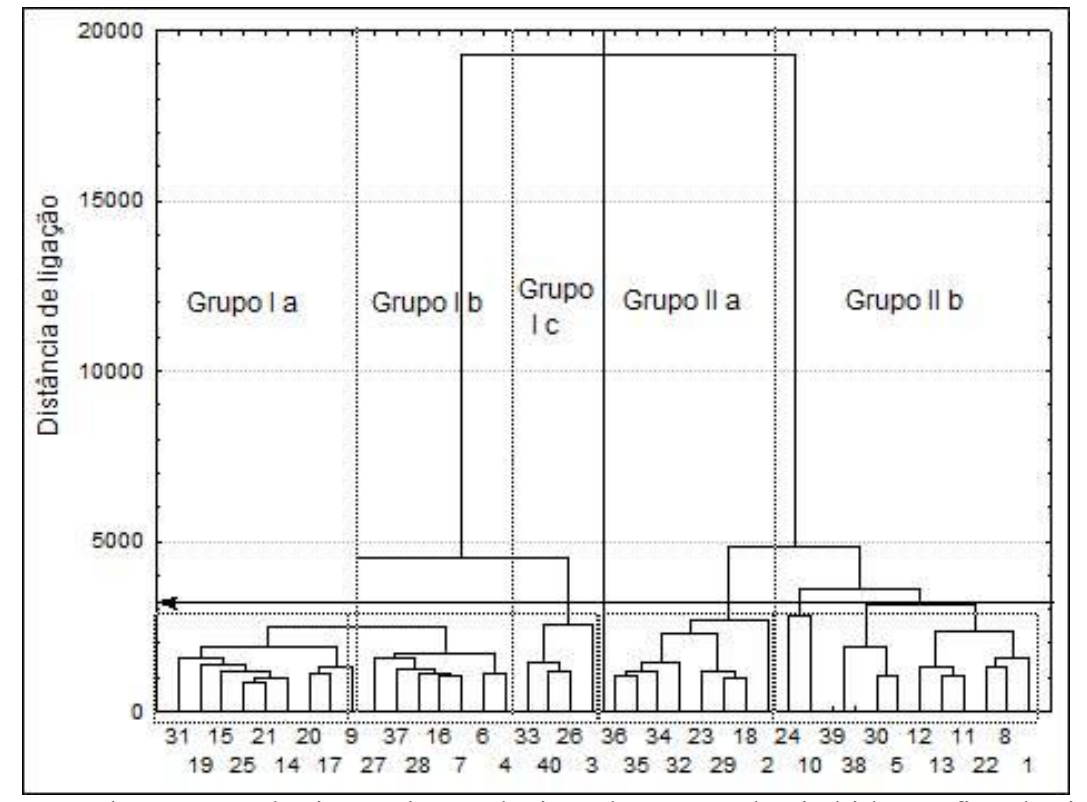

Figura 2 - Dendograma dos postos pluviométricos selecionados para a bacia hidrográfica do rio Piquiri - Paraná.

A figura 2 representa a espacialização dos postos pluviométricos, a altitude da área de estudo e os grupos homogêneos pluviométricos. Os postos pluviométricos do grupo homogêneo I ( $a, b$ e c ) concentram-se no setor centro-norte e oeste da bacia hidrográfica do rio Piquiri, com as altitudes mais baixas da área de estudo, predominantemente entre 200 a 500 metros, enquanto que os postos do grupo homogêneo II (a e b) localizam-se no setor centro-sul da bacia, com as altitudes mais elevadas, variando entre 500 a $1.000 \mathrm{~m}$.

Portanto, pode-se inferir que os padrões dos agrupamentos dos postos pluviométricos acompanham a altitude da bacia hidrográfica, pois nota-se a distinção dos grupos homogêneos I e II consonante às classes de altitude. A partir da associação entre distribuição espacial dos postos pluviométricos, seus respectivos grupos homogêneos e a altitude, observa-se que o relevo é um dos aspectos mais preponderantes para o controle da distribuição espacial dos totais anuais médios de precipitação pluvial na bacia hidrográfica do 


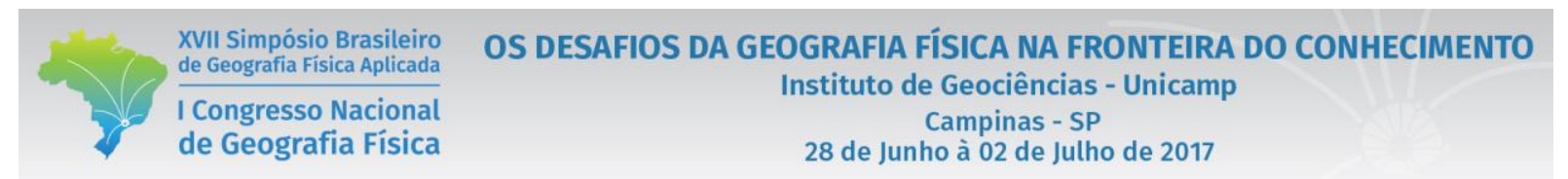

rio Piquiri, concordantes com os resultados obtidos para outras bacias hidrográficas, conforme obtiveram Andrade e Nery (2003), Baldo (2006), Chierice e Landim (2014) e Terassi et al. (2016).



Figura 3 - Grupos pluviométricos homogêneos para a bacia hidrográfica do rio Piquiri - Paraná.

Os grupos homogêneos Ia, Ib e Ic com altitudes médias entre 383 a 482 metros apresentam precipitação média anual variando entre 1.569 a $1.716 \mathrm{~mm}$. O grupo homogêneo Ia, de maiores altitudes e no setor setentrional deste grande grupo homogêneo demonstra o papel desempenhado pela orografia para a ocorrência de maiores totais de chuva na escala anual, ao passo que os grupos homogêneos Ib e Ic possuem a menor altitude média e por localizarem-se a noroeste, conforme descrevem Cavaglione et al. (2000) e Wrege et al. (2016) apresenta maiores similaridades com o clima tropical (Figura 3).

Os grupos homogêneos IIa e IIb apresentam altitudes entre 636 a 797 metros e precipitação anual média entre 1.885 e $2.034 \mathrm{~mm}$, evidenciando diretamente o efeito da orografia para a ocorrência de maiores totais de pluviosidade. O grupo homogêneo IIb localiza-se predominantemente no setor ao sul da área de estudo, que além da influência mais significativa do clima subtropical do Brasil Meridional (NERY, 2006; 
XVII Simpósio Brasileiro

de Geografia Física Aplicada

I Congresso Nacional

de Geografia Física
OS DESAFIOS DA GEOGRAFIA FÍSICA NA FRONTEIRA DO CONHECIMENTO

Instituto de Geociências - Unicamp

Campinas - SP

28 de Junho à 02 de Julho de 2017

NERY; CARFAN, 2014; APARECIDO et al., 2016), corresponde ao setor de maior altitude e que apresenta a maior influência da orografia para a geração das chuvas da área em estudo (Figura 3).

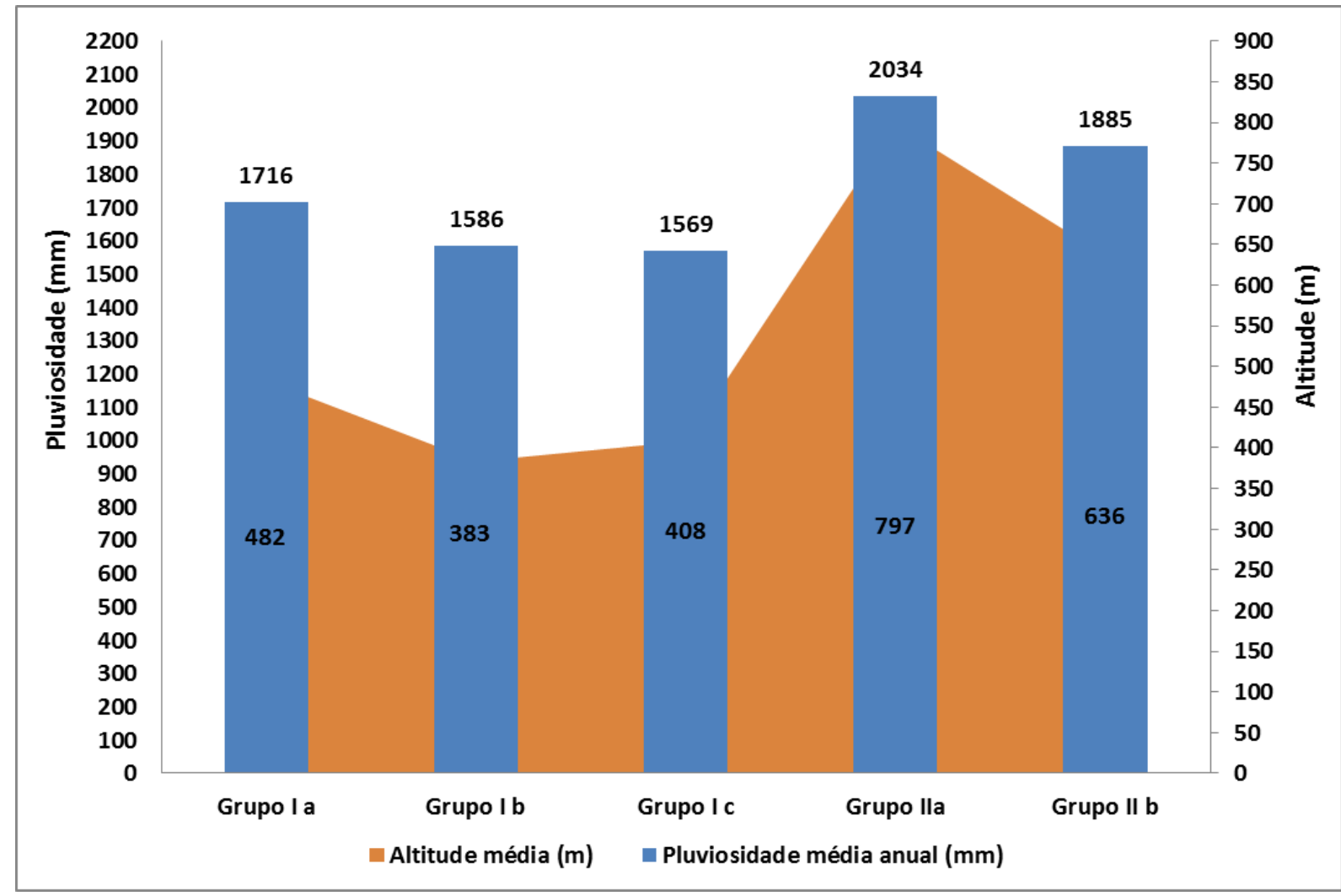

Figura 3- Pluviosidade média anual $(\mathrm{mm})$ e altitude média dos grupos homogêneos da bacia hidrográfica do rio Piquiri - Paraná.

A bacia hidrográfica do rio Piquiri situa-se em uma região de transição entre dois grandes tipos climáticos e, portanto, as características do regime de distribuição de chuvas estão condicionadas aos tipos de estados atmosféricos inerentes a esses domínios climáticos. De modo geral, a distribuição mensal em toda área de estudo caracteriza-se pela diminuição da precipitação média nos meses de julho e agosto, e um aumento durante a primavera e o verão, entre os meses de setembro a março, características próprias ao regime de precipitação no setor sul /sudeste da América do Sul (REBOITA et al., 2010).

O mês de outubro é o mais chuvoso para o grupo homogêneo II, o que delimita a maior proximidade com o clima subtropical (WREGE et al., 2016), posto que os totais pluviométricos mais elevados para este mês é uma característica própria este domínio climático, devido à combinação das entradas constantes dos sistemas polares com o aquecimento da superfície (TOMMASELLI et al., 2004). Por sua vez, o mês de dezembro é o mais chuvoso para o grupo homogêneo I, demonstrando uma maior proximidade ao regime 




de chuvas do clima tropical, que normalmente apresenta os maiores totais de pluviosidade entre os meses de dezembro a fevereiro (NIMER, 1989; SILVA et al., 2015).

O mês de agosto é o mais seco para todos os grupos homogêneos da área de estudo, com reduções mais significativas nos grupos homogêneos Ib e Ic, com médias inferiores a $70 \mathrm{~mm}$. A significativa redução das chuvas para os meses de agosto está relacionada à diminuição significativa dos processos convectivos em relação ao verão e a persistente atuação do sistema polar atlântico, o que ocasiona a predominância de maior estabilidade do tempo atmosférico (SILVA et al., 2006; ZANDONADI et al., 2015).

O mês de maio se destaca como um dos meses mais chuvosos do ano, uma vez que se trata de um período de transição entre estações e, conforme descreve Baldo (2006), a entrada mais frequente dos sistemas polares para o interior da região Sul do Brasil ao se chocarem com os sistemas atuantes mais aquecidos, como a massa tropical atlântica ou continental, ocasionam elevados valores de pluviosidade em comparação com os meses de abril e junho, principalmente para o grupo homogêneo II.



Figura 4 - Distribuição mensal da pluviosidade média mensal para os grupos homogêneos da bacia hidrográfica do rio Piquiri - Paraná. 


\section{Considerações finais}

Mostrou-se que a área em estudo apresenta duas regiões pluviométricas distintas. O grupo homogêneo I apresenta menores totais pluviais devido às menores altitudes e, com uma sazonalidade marcada, obteve as maiores diferença entre os meses menos chuvosos e os mais chuvosos, característica própria ao clima tropical do Brasil Central. O grupo homogêneo II mostrou os totais pluviométricos mais elevados, o que se deve a combinação de maiores altitudes e a predominância do clima subtropical do Brasil Meridional, condição destacada pela homogeneidade da distribuição do regime de chuvas.

A aplicação da análise de agrupamento e a espacialização dos postos pluviometricos sobreposto ao mapa hipsométrico mostrou a relação entre a distribuição dos totais de pluviosidade com a altitude e a associação com a localização dos grupos homogêneos permitiu delimitar as prováveis áreas de maior influência de dois grandes domínios climáticos.

\section{Agradecimentos}

Os autores agradecem ao Conselho Nacional de Desenvolvimento Científico e Tecnológico $(\mathrm{CNPq})$ e à Coordenação de Aperfeiçoamento de Pessoal de Nível Superior (CAPES) pela concessão das bolsas de estudos de Doutorado e à Bolsa de Produtividade em Pesquisa do CNPq - Nível 1D (303676/2013-2).

\section{Referências}

ÁlVARES, C. A.; STAPE, J. L.; SENTElHAS, P. C.; DE MORAES GONÇALVES, J.L.; SPAROVEK, G. Köppen's climate classification map for Brazil. Meteorologische Zeitschrift, Berlin, v. 22, n.6, p.711-728, 2013.

ANDRADE, A. R.; NERY, J. T. Análise da precipitação pluviométrica diária, mensal e interanual da bacia hidrográfica do Rio Ivaí, Brasil. Investigaciones Geográficas. Cidade do México, v.52, n.1, p.7-30, 2003.

APARECIDO, L. E. O; ROLIM, G. S.; RICHETTI, J.; SOUZA, P. S.; JOHANN, J. A. Köppen, Thornthwaite and Camargo climate classifications for climatic zoning in the State of Paraná, Brazil. Ciência e Agrotecnologia (Online), Lavras, v.40, n.4, p. 405-417, 2016.

BALDO, M.C. Variabilidade Pluviométrica e a Dinâmica Atmosférica da Bacia do Rio Ivaí. 2006. 153 f. Tese (Doutorado). Programa de Pós-Graduação em Geografia. Universidade Estadual Paulista "Júlio de Mesquita Filho", Presidente Prudente, 2006.

BRITO, T. T.; OLIVEIRA JÚNIOR, J. F. de; LYRA, G. B.; GOIS, G.; ZERI, M. Multivariate analysis applied to monthly rainfall over Rio de Janeiro state, Brazil. Meteorology and Atmospheric Physics, Berlin, v. 128, n.5, p. 1$10,2016$.

CAVIGLIONE, J. H.; KIIHL, L. R. B.; CARAMORI, P. H.; OLIVEIRA, D. Cartas climáticas do Paraná. Londrina: IAPAR, 2000. CD ROM.

CHIERICE, R.A.F.; LANDIM, P.M.B. Variabilidade espacial e temporal de precipitação pluviométrica na bacia hidrográfica do rio Mogi Guaçu. Geociências, São Paulo, v.33, n.1, p.157-171, 2014.

CORREA, M.G.G. Distribuição espacial e variabilidade da precipitação pluviométrica na bacia do rio Piquiri PR. 2013.102f. Dissertação (Mestrado). Programa de Pós-Graduação em Geografia Física, Universidade de São Paulo, São Paulo, 2013. 
CORREA, M. G. G.; TERASSI, P. M. B.; GALVANI, E. Efeito orográfico: estudo comparativo nas bacias hidrográficas do rio Piquiri e Pirapó - PR. In: XII Simpósio Brasileiro de Climatologia Geográfica. Variabilidade e susceptibilidade climática: implicações ecossistêmicas e sociais. Goiânia, p.1986-1997, 2016.

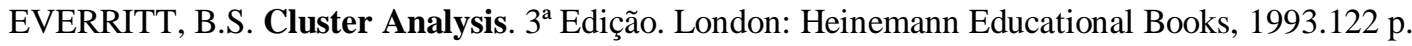

FREITAS, J.C.; ANDRADE, A.R.S.; BRAGA, C.C.; GODOI NETO, A.H.; ALMEIDA, T.F. Análise de agrupamento na identificação de regiões homogêneas de índices climáticos no Estado da Paraíba, Brasil. Revista Brasileira de Geografia Física, Recife, v.6, n.4, p.732-748, 2013.

FRITZSONS, E.; MANTOVANI, L. E.; WREGE, M. S.; CHAVES NETO, A. Análise da pluviometria para definição de zonas homogêneas no Estado do Paraná. RA'E GA: o Espaço Geográfico em Análise, Curitiba, v.23, n.1, p.555-572, 2011.

ITCG (Instituto de Terras, Cartografia e Geodésia). Produtos Cartográficos. Disponível em: $<$ http://www.itcg.pr.gov.br/modules/conteudo/conteudo. php?conteudo=47. Acesso em 10 de fevereiro de 2017.

KELLER FILHO, T.; ASSAD, E.D.; LIMA, P.R.S.R. Regiões pluviometricamente homogêneas no Brasil. Revista Brasileira de Pesquisa Agropecuária, Brasília, v.40, n.4, p.311-322, 2005.

LEIVAS, J.F.; BERLATO, M.A.; FONTANA, D.C. Risco de deficiência hídrica decendial na metade sul do Rio Grande do Sul. Revista Brasileira de Engenharia Agrícola e Ambiental. Campina Grande, v.10, n.2, p.397- 407, 2006.

MARTIN, T. N.; NETO, D. D.; VIEIRA JUNIOR, P. A.; MANFRON, P. A. Homogeneidade espaço temporal e modelos de distribuição para a precipitação pluvial no estado de São Paulo. Revista Ceres, Viçosa, v.55, n.5, p.476481, 2008

MELO JÚNIOR, J. C. F.; SEDIYAMA, G. C.; FERREIRA, P. A.; LEAL, B. G. Determinação de regiões homogêneas quanto à distribuição de freqüência de chuvas no leste do Estado de Minas Gerais. Revista Brasileira de Engenharia Agrícola e Ambiental, Campina Grande, v.10, n.2, p.408-416, 2006.

NASCIMENTO, F.C.A.; ARAÚJO, F.R.C.D.; BRAGA, C.C.; COSTA, E.V.S. Análise dos padrões espaciais e temporais da precipitação no Estado do Maranhão. Revista Brasileira de Geografia Física, Recife, v.8, n.2, p.422430, 2015.

NERY, J. T. Dinâmica climática da região Sul do Brasil. Revista Brasileira de Climatologia, Curitiba, v.1, n.1, p. 61-75, 2006.

NERY, J.T.; CARFAN, A.C. Re-analysis of pluvial precipitation in southern Brazil. Atmosféra, Cidade do México, v.27, n.2, p.103-114, 2014.

NIMER. E. Climatologia do Brasil. 2ªEdição. Rio de Janeiro: Instituto Brasileiro de Geografia e Estatística, 1989. $421 \mathrm{p}$.

REBOITA, M.S.; GAN, M.A.; ROCHA, R.P.; AMBRIZZI, T. Regimes de precipitação na América do Sul. Revista Brasileira de Meteorologia, São Paulo, v.25, n.2, p.185-204, 2010.

SANTOS, W.A.; ARAÚJO, H.M. Clima e condições meteorológicas da sub-bacia hidrográfica do rio Contiguiba SE. Boletim de Geografia, Maringá, v.31, n.1, p.41-52, 2013.

SILVA, C. B.; SANT'ANNA NETO, J. L.; TOMMASELLI, J. T. G.; PASSOS, M. M. Dinâmica atmosférica e análise geoestatística do clima na área de integração paisagística 'Raia Divisória' SP/PR/MS: uma proposta de tipologia climática. Revista Brasileira de Climatologia, Curitiba, v.2, p.53-70, 2006.

SILVA, W.L.; DERECZYNSKI, C.; CHANG, M.; FREITAS, M.; MACHADO, B.J.; TRISTÃO, L.; RUGGERI, J. Tendências observadas em indicadores de extremos climáticos de temperatura e precipitação no Estado do Paraná. Revista Brasileira de Meteorologia, São Paulo, v.30, n.2, p.181-194, 2015.

SILVESTRE, M. R.; SANT'ANNA NETO, J. L.; FLORES, E. F. Critérios estatísticos para definir anos padrão: uma contribuição à Climatologia Geográfica. Revista Formação, Presidente Prudente, v.2, n.20, p.23-53, 2013. 




TEODORO, P.E.; DE OLIVEIRA-JÚNIOR, J.F.; DA CUNHA, E. R.; CORREA, C. C. G.; TORRES, F. E.; BACANI, V. M.; GOIS, G.; RIBEIRO, L. P. Cluster analysis applied to the spatial and temporal variability of monthly rainfall in Mato Grosso do Sul State, Brazil. Meteorology and Atmospheric Physics, Berlin, v.128, n.6, p.197-209, 2015.

TERASSI, P. M. B.; SILVEIRA, H.; GRAÇA, C. H. Regiões pluviométricas homogêneas e a erosividade das chuvas na unidade hidrográfica Pirapó, Paranapanema III e IV-Paraná. Caderno de Geografia, Belo Horizonte, v.26, n.46, p.507-521, 2016.

TOMMASELLI, J. T. G.; BALDO, M. C.; SILVEIRA, H. Caracterização pluviométrica da mesorregião geográfica centro ocidental paranaense. In: VI Simpósio Brasileiro de Climatologia Geográfica, Aracaju, p.1-9, 2004.

VILLELA, S. M.; MATTOS, A. Hidrologia aplicada. São Paulo: McGraw-Hill do Brasil, 1975. 245p.

WARD, J.H. Hierarquical grouping to optimize an objective function. Journal of the American Statistical Association, v.58, n.301, p.236-244, 1963.

WREGE, M. S.; FRITZSONS, E.; CARAMORI, P. H.; RICCE, W.S.; RADIN, B.; STEINMETZ, S.; REISSER JÚNIOR, C. Regiões com similaridade de comportamento hídrico no Sul do Brasil. RA'E GA: o Espaço Geográfico em Análise, Curitiba, v.38, p.363-382, 2016.

ZANDONADI, L. As chuvas da bacia do rio Paraná, Brasil: Um estudo do ritmo climático e algumas considerações sobre a vazão hídrica. 2013. 206f. Tese (Doutorado). Programa de Pós-Graduação em Geografia, Universidade Estadual Paulista "Júlio de Mesquita Filho", Rio Claro, 2013.

ZANDONADI, L.; ACQUAOTTA, F.; FRATIANNI, S.; ZAVATTINI, J. A. Changes in precipitation extremes in Brazil (Paraná River Basin). Theoretical and Applied Climatology, v. 119, n.1, p. 741-756, 2015. 